This is an electronic reprint of the original article. This reprint may differ from the original in pagination and typographic detail.

Author(s): Hongell, Timo; Kivelä, Ilkka; Hakala, Ismo

Title: Wireless Strain Gauge Network : Best-Hall Measurement Case

Year: $\quad 2014$

Version:

Please cite the original version:

Hongell, T., Kivelä, I., \& Hakala, I. (2014). Wireless Strain Gauge Network : Best-Hall Measurement Case. In W. Seah, S. Maag, \& J. Gubbi (Eds.), Proceedings of IEEE ISSNIP 2014 : IEEE Ninth International Conference on Intelligent Sensors, Sensor Networks and Information Processing (pp. 1-6). IEEE.

https://doi.org/10.1109/ISSNIP.2014.6827665

All material supplied via JYX is protected by copyright and other intellectual property rights, and duplication or sale of all or part of any of the repository collections is not permitted, except that material may be duplicated by you for your research use or educational purposes in electronic or print form. You must obtain permission for any other use. Electronic or print copies may not be offered, whether for sale or otherwise to anyone who is not an authorised user. 


\title{
Wireless Strain Gauge Network - Best-Hall Measurement Case
}

\author{
Timo Hongell, Ilkka Kivelä, Ismo Hakala \\ University of Jyväskylä / Kokkola University Consortium Chydenius \\ P.O.Box 567, FI-67701, Kokkola \\ Email: first_name.last_name@chydenius.fi
}

\begin{abstract}
Wireless sensor networks can be used to perform structural monitoring. Strain gauges, which can be glued on or integrated to the observed material, are the most common nondestructive sensing elements for measuring surface strain. Wireless strain gauge measurement system makes strain measurements more flexible while opening new targets of application in addition to those that traditional wired strain measuring systems can offer. We have implemented a long term strain measurement in a PVC-covered hall using wireless sensor network. In this paper we evaluate the functionality and suitability of the developed wireless system for this kind of monitoring. The paper discusses the experiences gained in the development and utilization of a wireless strain gauge measurement system. The calibration of measurement system as well as energy consumption issues are also brought under observation along with the reliability of communication aspects in the described measurement case.
\end{abstract}

\section{INTRODUCTION}

In recent years, study of wireless sensor networks (WSN) has become a rapidly developing research area. WSN is a set of wireless sensor nodes. Each of its nodes measures a physical value, using selected sensor probes, and sends the value to a database through specific sink or gateway nodes. WSNs are widely used in civil and industrial applications such as smart home or environment monitoring [1]-[3]. Compared to traditional sensing methods, WSN technology offers some additional benefits: wide areas can be covered with inexpensive, energy-efficient battery-powered devices, which make long-term monitoring and real time access to measuring data possible. Often the nodes of WSN are able to self-configure themselves, which enables quick and easy system deployment.

Strain gauge sensors must be attached afterwards or integrated to the measured material during manufacturing. When compared to traditional wired strain gauge systems, the wireless solution provides more adaptivity and flexibility to the measurements. Wired systems require that the installation cables are connected from the measurement location to the end device. The extra cables may cause interference to the measurements, and they also make the installation process more complicated and increase the costs. Depending on the measurement environment and the radio solution used, the wireless systems have transmission ranges from few meters up to few hundred meters. The advantage of a wireless system compared to a wired one is that it can operate in different difficult-to-reach surroundings such as sealing and wall structures in buildings and rotating objects, which are difficult or even impossible to effectively measure using wired systems. Wireless solutions also make strain gauge embedding to the measured material feasible, since the short connection wires to the nodes can be installed afterwards. The networks are flexible and can be easily widened if an additional measurement point is required. Wireless solutions can be used for temporary network setups, where the measurement data gathering is done only for a known period of time and where the measurement system is to be removed afterwards. Since the nodes communicate together and are time synchronized, different types of measurement data can be used together to analyze the strain values. For example, in measurements of PVC-covered fabric, temperature, wind and moisture may directly affect the strain measurement values.

This paper discusses the design and implementing of the $3^{\text {rd }}$ development version of a strain gauge measurement system for a wireless IEEE802.15.4- standard CiNet network and experiences of a measurement case where the system is used. The network can automatically configure and send data after the node deployment. The system is remotely monitored and controlled using a web based application. The main focus of the paper is on describing, designing and evaluating the battery powered strain gauge sensor system in a real deployment in a PVC-covered hall, manufactured by Best-Hall, which is the market leader in the product development and quality of halls with demanding steel frameworks [4]. We consider the wireless systems reliability and also how does the wireless systems installation and adaptability manage and suits to the challenging conditions.

The paper is organized as follows: First we provide a brief description of some related research and describe the technologies used. Section III presents the CiNetStrain system created for this research and the node calibration for the measurement case. Section V describes the measurement case, the network setup and the strain gauge measurements done. Finally, some evaluation of the system, experiences about the measurements and future development are discussed.

\section{RELATED WORK}

Strain gauge measurements are widely used in different systems and deployments, but the study of those measurements in a wireless environment is quite marginal. The general features of wireless strain gauge system requirements and system design are discussed in [5]. Bielen et al. have developed a WMS 80 based wireless measurement system for rotating structures that can support strain gauges [6]. Their study focuses mainly on building a complete system; the basic data gathering procedures or the initial calibration with any reference device of the nodes are not explained. In [7] Jian et al. describe the development of an IEEE802.15.4 based 
wireless strain mapping system, where they use commercial WSN RF modules and focus mainly on strain mapping. Some new commercial solutions have been developed, but they are relatively costly and limited. One wireless solution is the MicroStrain SG-Link ${ }^{\circledR}-$ XXRS $^{\mathrm{TM}}$ Wireless Strain Node developed by MicroStrain Inc [8].

The basics of strain gauge measurements are explained in detail in the related papers, but some interesting features and constrains of wireless strain gauge systems are discussed only briefly or not at all taken into consideration. Those features include e.g. the sensor platform design, the used measurement protocol and also the systems' true energy consumption that is very important for wireless systems. Our solution advantages to the commercial products are it's flexibility and versatility, as many different measurement sensors can be included to one node, and the fact that all measured data can be preprocessed in the node. The wireless nodes typically operate as a network and the whole measurement area can be analyzed as one entity, which enables new possibilities to different application approaches and system solutions.

In this paper, we also explain in detail our solution on how to procedure wireless strain measurements. The CiNetStrain system differs from the referenced systems by its scalability and capability to support multiple different sensor solutions at the same time. Additional nodes can easily be introduced to the network, and the data produced by them can be analyzed together with other nodes. This paper also describes the data gathering procedure, calibration and the energy consumption of the nodes. Moreover, there is a review of the measurement results and evaluation of the networks reliability and suitability to challenging measurement conditions. Throughput and LQI values of the network are used in the evaluation.

\section{CinetStrain System}

The third development version of the wireless CiNetStrain system was built on the CiNet network nodes [2] [9], see Figures 1 and 2. CiNet is a research and development platform for the WSN implemented in Kokkola University Consortium Chydenius, University of Jyväskylä. The hardware in the node is specially designed for WSNs and consists of inexpensive standard off-the-shelf components. The CiNet node includes all the basic components necessary for WSNs.

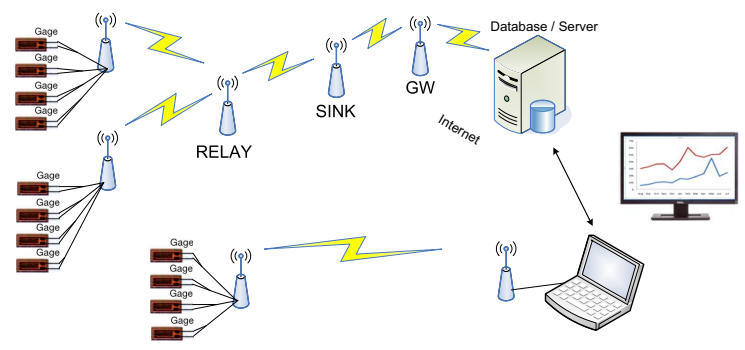

Fig. 1. The typical system structure of CiNetStrain network.

The CiNet WSN includes the measurement, relay and sink nodes that can communicate and transmit data among themselves, using wireless radio transmitters. The nodes are able to automatically configure the network structure and, therefore, it is simple to add more nodes to the measurement

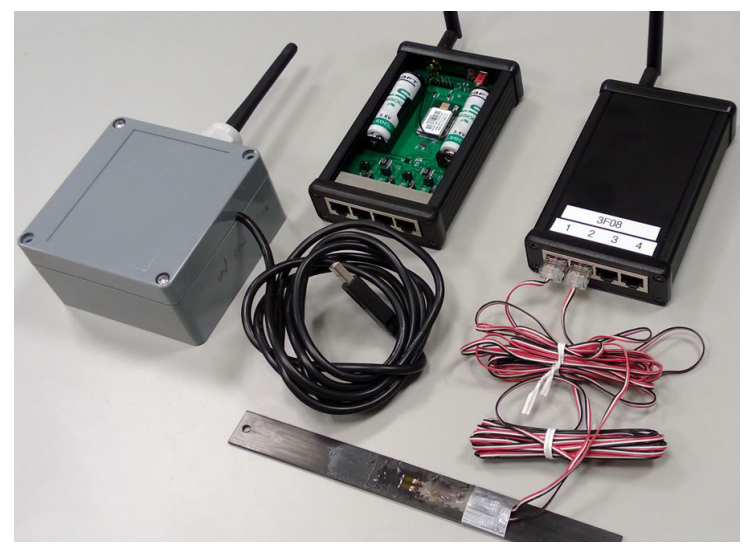

Fig. 2. Wireless strain measurement devices (SINK and nodes) and attached strain gauges.

network. Typically the sink node gathers and transmits the measurements to the gateway, which transmits the data to a specified database. Database usually runs on a Apache web server. This can be done using e.g. GPRS transmission or direct USB, Ethernet or serial cable connection from a sink to the computer. The data transmission between the nodes can be multi-hop or single-hop based. Multi-hop transmission allows relaying nodes to be used when the distances between the measurement and the sink node are longer. The measurement network used is synchronized and the nodes use CiNet crosslayer protocol stack that allows data sharing, without additional messaging, between different protocol layers. The network basically operates in the way depicted in more detailed in [10].

\section{A. CiNetStrain Nodes}

The nodes use $2.4 \mathrm{GHz}$ Jennic IEEE802.15.4 radio modules [11] and can work seamlessly in the CiNet network. The nodes are typically powered with two industrial 3.6 volt AAbatteries, but they can also be directly connected to an electric power network, see Table I. The strain gauges, which are the main sensors of this system, are attached to the nodes with the gauges' lead wires and a RJ45 connector. The nodes can be configured to measure strain values at user-defined time intervals. For example, strain values can be measured continuously, or the nodes can be defined to go to sleep mode and wake up to measure strain values on defined intervals. The measurement intervals can be remotely set to the nodes, and the nodes' strain measurement can also be remotely zeroed.

TABLE I. CINETSTRAIN NODE INFORMATION

\begin{tabular}{|c|c|}
\hline Wireless MCU & Jennic JN5148 \\
\hline Current consumption & Active: $17.37 \mathrm{~mA}$ Sleep: $16 \mu \mathrm{A}$ \\
\hline Analog to Digital Converter & Four channel 16 bit ADC \\
\hline Battery & 2X AA 2600 mAh, 3.6 V (Li-SOCl2) \\
\hline External Power & $2.7 \mathrm{~V}-3.3 \mathrm{~V}$ \\
\hline Casing Dimensions & $120 \times 78 \times 27(\mathrm{~mm})$ \\
\hline
\end{tabular}

1) Strain Gauges: The nodes can be equipped with various kinds of sensors to measure, e.g., temperature, humidity, noise, vibration and strain. In this study, we are using strain gauge sensors. A strain gauge is a sensor whose resistance varies with applied force. It converts force, pressure, tension, weight, etc., into a change in electrical resistance, which can then be measured. When external forces are applied to a stationary object, 
stress and strain result. Stress is defined as the object's internal resisting forces, and strain is defined as the displacement and deformation that occurs in the object.

The CiNetStrain nodes (see Fig. 2) are modified from the basic CiNet nodes by equipping them with four Wheatstone bridge resistor setups using precision resistors and applying required electronics and connectors for the strain gauges. The strain gauges measure resistance changes, caused by the physical changes in the element, but the resistance changes are very small. Wheatstone bridge is an electric circuit suitable for detection of very small resistance changes. It is typically used to enhance the measured resistance changes of a strain gauge. The four bridges allow the nodes to have four independent measurement channels that all can measure strain values.

\section{B. Strain Data Sampling}

In this study case, the strain nodes measure 20 raw-data ADC samples to produce one strain measurement. The rawdata samples are taken at $2 \mathrm{~ms}$ intervals and are sorted from the smallest to the largest. The largest and the smallest values are ignored to reduce false sampling. The average of the remaining 18 values is computed and sent to a server to be stored in a database. The nodes' final output shown on the graphical display is the average of the user-defined time interval. This double averaging is done to minimize the noise and errors in the ADC raw-data measurements.

The $3^{r d}$ version of the strain node has four different strain measurement channels, all of which can be used virtually at the same time, as one node can support four strain gauges. The node does first the ADC-sampling for channel 0 and then for channel 1,2 and 3. While this takes place, the measurement bridges are not on at the same time, which reduces the power consumption. The time difference between each of the sampled channels is $15 \mathrm{~ms}$. Depending on the measurement case and application, the requirements for the time intervals between the measurements change. For the measurements of this study, the data sampling cycle is defined as shown in Fig. 3.

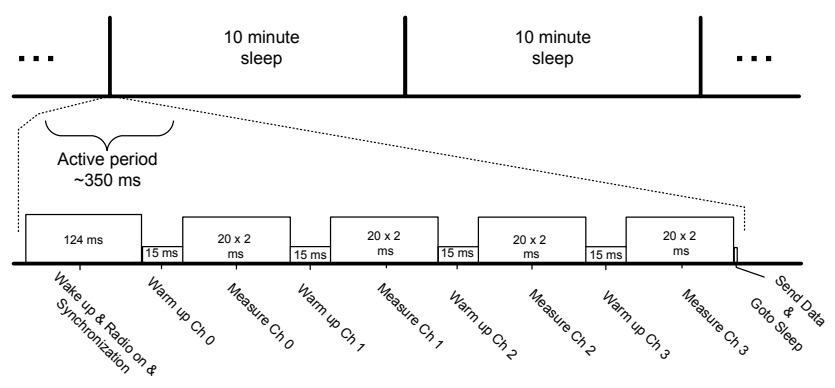

Fig. 3. CiNetStrain data sampling cycle.

\section{CiNetStrain User Interface}

The CiNetStrain system is configured to work seamlessly with the CiNet Web App application (see Fig. 7). This application is the controlling software that is used to visualize and control the collected data. It is basically a web based online program that can be used remotely with a PC or laptop computer and also with tablet and other mobile devices. The application reads and displays the measured values in a graphical user interface window. Strain data for every channel of each strain node can be displayed with different line colors. With the software, it is possible to define the different time intervals for which all the measured data can be retrieved from the server and saved to a csv-file for later analysis.

\section{Best-Hall Node CALIBRATION AND ENERGY CONSUMPTION}

Operational validation of the strain nodes has been done and was presented in [12], where the system is compared to a wired commercial strain gauge system. The conclusion arrived at in that paper is that the CiNetStrain system can produce reliable and accurate strain values.

The calibration of the strain nodes for the Best-Hall measurement case was done in two parts using a large fabricstretching bench, see Fig. 4. The calibration device and the sample materials were provided by Best-Hall. The strain gauges were attached to a sample PVC-covered fabric, and, using a pulley, the fabric was stretched. At first (case A), the fabric was attached from both of its ends.

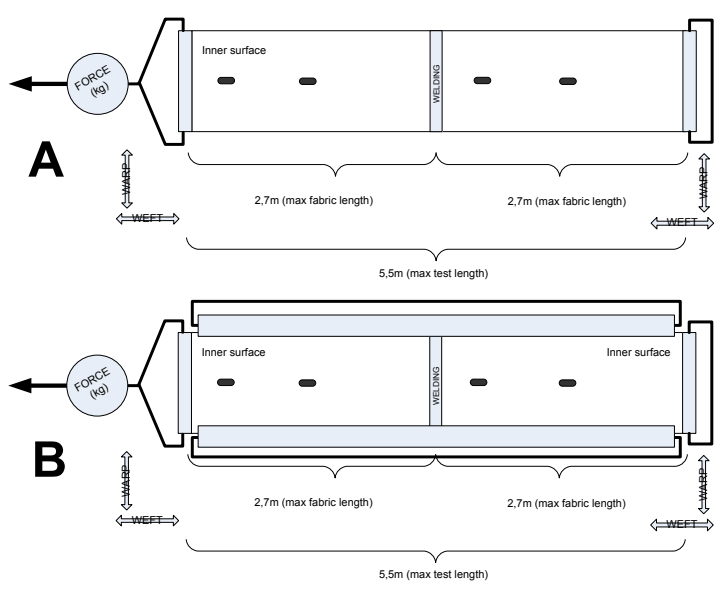

Fig. 4. Calibration of the CiNetStrain system.

The ADC value of the node and the real stretch, with different forces, of the fabric were recorded. Based on these results, the linear conversion equation can be defined as

$$
y=0,011 * x-0,144,
$$

where $x$ is the input in ADC values and $y$ is the output as millimeter to meter stretch value. For the second test (case B), the fabric was attached to the bench form all of its four sides. Based on the second test results, the linear conversion equation can be defined as

$$
y=0,009 * x-0,032,
$$

where again $x$ is the input in ADC values and $y$ is the output as millimeter to meter stretch value.

Based on the two different calibrations, we can simplify the overall ADC to millimeter to meter conversion formula by making it the average of the two calibrations

$$
y=0,01 * x
$$

where again $x$ is the input in ADC values and $y$ is the output as millimeter to meter stretch value. This simplification can 
be done, since the true installations of the fabric are a kind of combination of these two setups. Using Equation 3, the wireless nodes are linearly calibrated to produce micro-strain values, equal to the true measured stretch. The equation is given for the application, and it converts the ADC values to micro-strain values. This conversion is done to make the measured strain values more reasonable for the viewer.

\section{A. Zero-gauges}

Zero-gauge compensation is typically done to neglect the temperature effect on the strain measurements. Strain gauges which are connected to a material similar to the materials measured but which are not put under tension are called zerogauges. This way, only the temperature affects the material, and its effect can then be taken into consideration. For the other strain measurements, it is possible to separate the strain values resulting from the temperature changes and other physically caused tensions (snow, wind, ext.).

\section{B. Energy Consumption}

The wireless CiNetStrain nodes are tested in laboratory measured tests to find out the energy consumption and the practical battery life for the nodes. The idea of the system is to enable long-term measurements that are typical and practical in strain gauge deployment scenarios. This kind of application can be e.g. structural monitoring of a bridge or a building or, as in this case, a Best-Hall-type measurement.

In the laboratory test, the node's current consumption is measured using oscilloscope in a typical measurement scenario over a small resistor (see e.g. [13]). The current consumption is calculated from ten repetitions of the measurement results of two different CiNetStrain nodes. The different phases of the duty cycle are shown in Fig. 3. For the current measurement study, the measuring cycle is set to 10 seconds. The power supply is by typical AA-batteries. The wake-up and synchronization of the node take about $125 \mathrm{~ms}$, the measuring and transmitting about $250 \mathrm{~ms}$, and for the rest of the time the node will be in sleep mode, see Fig. 3. Based on the measurements, the current consumption of one node during a strain measurement can be defined using the nodes' active and sleeping mode consumption, see Fig. 5. The active period is about $350 \mathrm{~ms}$ and is divided into two phases. In the first phase, the node wakes up, turns the radio on and gets synchronized with average current consumption of $21.3 \mathrm{~mA}$. The second phase consists of the measurement and transmission of the data, and the average current consumption is about $15.2 \mathrm{~mA}$. The total active period current consumption is $17.37 \mathrm{~mA}$. The sleeping mode current consumption is about $16 \mu \mathrm{A}$, and the sleeping duration depends on the measurement scenario used.

When the nodes are equipped with two 3.6 volt $2600 \mathrm{mAh}$ industrial (Li-SOCl2)AA-batteries, the computational operation time of the nodes varies depending on the measurement cycle used. For example, with a one-second cycle (basically a real-time operation), the battery duration is over one month, a five-second cycle guarantees over half a year of operation time, a half-minute cycle gives about three years and cycles of oneminute and above will provide several years of computational operation time. These operation times are adequate for almost any kind of measurement scenario.

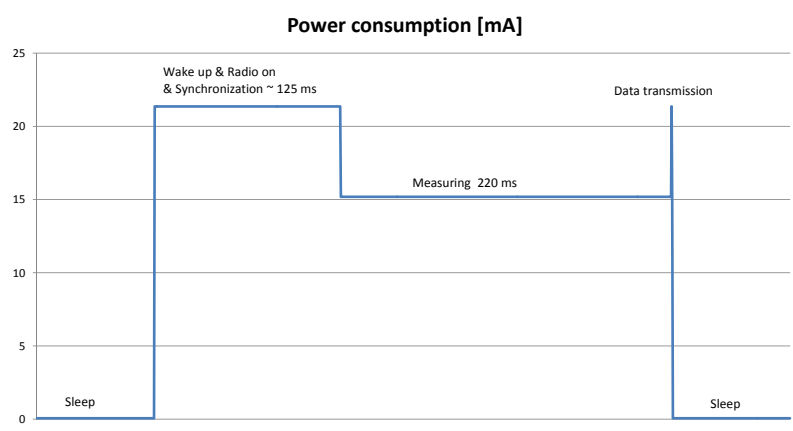

Fig. 5. Current consumption of the CiNetStrain node in one measurement.

\section{Strain Gauge Measurements for the BEST-HALL CASE}

We used Micro-Measurements' Model D4 Data Acquisition Conditioner [14] as a commercial measurement and reference device. The CiNetStrain nodes' output was validated with the D4 device before the installation. We also employed $120 \Omega$ strain gauges, which are KYOWA General Purpose KFG-5120-C1-11L110M3R strain gauges [15] that are suitable for PVC-covered materials.

In the PVC-covered hall, the strain gauges are attached to the fabric at different altitudes and locations (spots). Each measurement spot has two orthogonally (vertical and horizontal) placed strain gauges. One gauge is attached to the warp direction and one gauge is attached to the weft direction that measure the physical changes in the fabric. Four of the gauges are attached to the hall's steel frame. The strain gauges' lead wires are attached to the fabric and the structures and connected to the strain nodes. One node can support four gauges that cover two measurement spots. The lead wire lengths vary from three to ten meters, depending on the measurement spot location. We also deployed three zero-gauges at different altitudes to find out the altitudes' effect on the temperature and also on the strain measurement. The strain values at different altitudes (walls, ground and sealing) are compensated based on the zero gauges at the same altitude. After the installation procedure, all the strain nodes were simultaneously zeroed to the initial state prior to the measurements. The strain values recorded to the database during the measurements are compared to the zeroing state of the hall.

\section{A. Best-Hall Network}

In the Best-Hall case, the measurement network (see Fig. 6) includes one SINK-node, one node for measuring temperature and humidity, twelve strain measuring nodes, with four strain gauges and a commercial AIRMAR ${ }^{\mathrm{R}}$ WeatherStation ${ }^{\mathrm{R}}$ Instrument $150 \mathrm{WX}$. The weather station collects real-time weather data that includes temperature, humidity as well as wind speed and direction from outdoors and is time synchronized with the measurement network. The sink and the weather station are connected to a Raspberry Pi minicomputer gateway. The strain measuring nodes gather information from a $30 \mathrm{x}$ $20 \mathrm{~m}$ area. Because the measurement area and the measuring intervals are relatively small, the network topology selected is star topology, where the nodes are connected straight to the sink node. The usage of relay nodes is not needed, since the nodes can hear the sink node without them. In this setup 
scenario, since no multi-hop network is needed, the relayfree star topology also can reduce data transmission error probability and unreliability of the data transmission. The nodes are synchronized at every ten-minute's measurement period. Basically, in one hour the network produces about 300 different measurements that are transmitted to the sink node and then onwards to the database. All the collected information can be analyzed together using the CiNet Web App application.

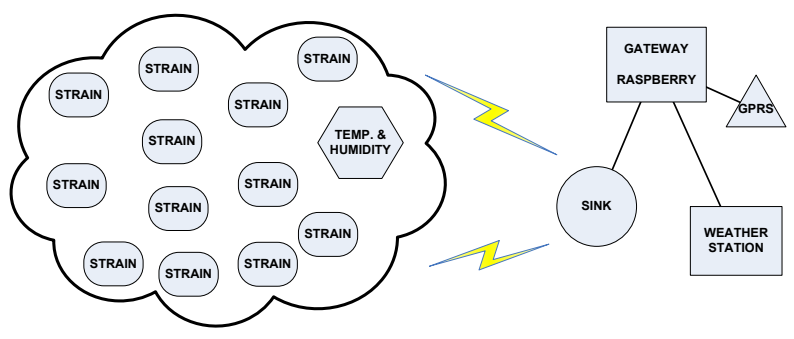

Fig. 6. Best-Hall measurement network (not in scale).

\section{B. Network statistics}

By the writing of this paper, the network has been gathering information roughly for eight months from the end of February 2013 to end of October 2013. During this period, the Raspberry Pi computer became jammed in a couple of occasions, but the wireless network was working all the time. Because the Raspberry Pi is used to control the data transmission through the GPRS-gateway, the data measured during Raspberry Pi's malfunctioning was not added to the database. The longest malfunction of Raspberry Pi happened during the Finnish winter holiday week, and the computer was repaired after that. Also, there were shorter Raspberry Pi jams. The days when the Raspberry Pi computer was out of action are excluded from the wireless strain network analysis. The following analysis is based on the measurement results over 220 days, during which period the network was operational full 24 hours in a day. During these days, each node took measurements once in every ten minutes with four channels. For one node, in a day, this leads to 144 measurement cycles and 576 measured and transmitted strain values. After each measurement cycle, one data packet that includes all four channels' measurement information is sent to the sink node. During the 220 days, a total of about 400000 data packets were transmitted. The total average throughput of the network nodes in that period was $97.26 \%$, which indicates a steady and reliable network. The throughput of each node is shown in Table II.

TABLE II. THE AVERAGE THROUGHPUT AND LQI VALUES OF THE BEST-HALL NETWORK.

\begin{tabular}{|c|c|c|c|c|c|}
\hline Node ID & throughput & LQI & Node ID & throughput & LQI \\
\hline 3F00 & $96.69 \%$ & 44.38 & 3F01 & $95.39 \%$ & 30.86 \\
\hline 3F02 & $95.45 \%$ & 31.10 & 3F03 & $97.23 \%$ & 41.91 \\
\hline 3F04 & $95.01 \%$ & 62.27 & 3F05 & $97.67 \%$ & 61.37 \\
\hline 3F06 & $98.72 \%$ & 61.91 & 3F07 & $99.55 \%$ & 97.49 \\
\hline 3F08 & $98.95 \%$ & 50.89 & 3F09 & $98.98 \%$ & 56.87 \\
\hline 3F10 & $95.40 \%$ & 26.15 & 3F11 & $97.85 \%$ & 57.78 \\
\hline 3F21 & $99.57 \%$ & 72.08 & Total & $97.26 \%$ & 53.47 \\
\hline
\end{tabular}

Overall, the nodes' throughput values are great. Four of the nodes (3F02, 3F03, 3F04 and 3F10) had a bit smaller values, which may be explained with node placements. These nodes were on the edges of the measurement area, and some steel bars had been on the line-of-sight to the sink node. The average link quality indicator (LQI) values indicate how strong the communications link is. LQI is a computed value, based on the received signal strength and on the number of errors received. LQI values of the nodes are in line with the throughput values.

\section{Measurement Results of the Application}

The main idea of the Best-Hall measurements is to easily monitor and visualize the PVC-fabrics behavior in different environmental conditions in real time. We measured six times in an hour, to meet once in an hour system requirement. Therefore, in the application point of view, data have been collected more than enough and the system functionality have been nearly $100 \%$. The strain values of the halls' fabric and steel frames can be analyzed together in real time. The strain measurements agree with the results of different nodes. One node's four channels strain values during one week are shown in Fig. 7. Since all the measurements are done in real time and are synchronized, collected environmental measurement data can be used together with the strain measurements that is one of the clear advantages of adaptive and extendable WSN system. Deeper analysis reveals that e.g. snow and wind condition changes can be noticed from the measured strain values. The measured hall can easily be remotely monitored using the web application and the data can be visualized in real time. In overall, the application met the system requirements.

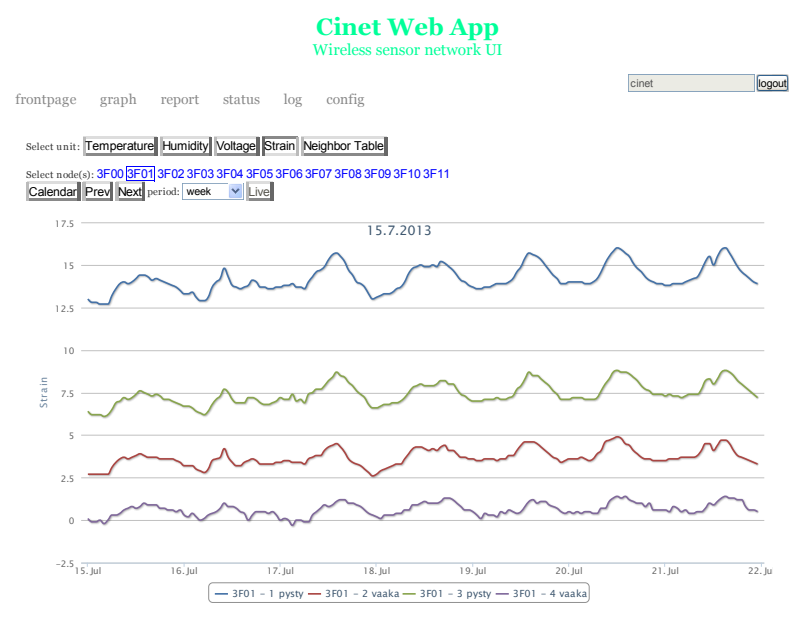

Fig. 7. Example strain values in CiNet Web App application.

\section{DISCUSSION}

The wireless CiNetStrain system passes the both operational and reliability evaluations. The network was relatively simple and free to install, and due to wireless operation more than few hundred meters of cabling was saved. The networks easy extendability and self configuration ability was tested, when a new measurement node was afterwards introduced to the system. After the installation and power switched on, it worked seamlessly with existing system. Battery-powered strain nodes proved to be a good solution. As relatively small amount of energy is consumed, none of the nodes have needed maintenance after about eight months of continuous operation. 
The hall has its own microclimate conditions that differ from the outside weather conditions, including temperature and humidity. The humidity in the hall gave rise to severe ice frost to the structures, that also affected the node installation and the battery operated nodes themselves. The system and its measurements are been monitored, controlled and visualized, in real time, successfully using a web based application. Several different sources of data have not shown to be any kind of problem, on a contrast, using data fusion with the strain and environmental measurements, snow and wind conditions can be linked with the changes in the strain values. The measured strain values are in line with the other measured variables. The whole wireless network itself was working fine. At first, the sink node, GPRS and the weather station were connected to the Raspberry Pi computer through a USB hub. Raspberry Pi had some problems in holding the USB port numbering for the USB hub if it got rebooted. Due to this, the data was not transmitted to the database in these occasions. The problems having been solved, the wireless CiNetStrain system, in general, was proven to be usable and reliable system for strain measurements. The wireless network is been operating really well with over $97 \%$ throughput value.

\section{CONCLUSIONS}

In this paper, we have presented and evaluated a working strain-based time-synchronized wireless CiNetStrain monitoring system that has proven itself to be used to diagnose structures and easily extended to large deployments at low cost. The strain nodes have low power consumption that is tested and noted in a real long term deployment, and with a typical setup the nodes can be operational over one year without a battery change. Thus, the presented system shows that the strain measurements can be done in a WSN, where the resources, such as energy, memory, computing capacity and even the price, are constrained. This indicates that wireless solutions make strain gauge embedding to the measured material feasible, also in large measurement scenarios as well as in temporary measurement cases.

The easily installable wireless network is automatically scalable, and additional nodes can easily be introduced to the network. The data produced by them can be analyzed together with the other nodes. The additional nodes can produce information that can be used to support the strain measurements, e.g. temperature and humidity. The system can be applied to a wide range of target types, and it is equipped with a multi-hop wireless communication capability. The strain sensor board using a Jennic radio that we have designed was proven as a reliable source of strain data. GUI-based monitoring software was developed to analyze and visualize the strain data. Thus, to monitor structural conditions, we have developed a fully functional and evaluated wireless system for measuring strain, wherefore the measured data can be directly used to trigger maintenance events and report alerts in real time scenarios.

The Best-Hall measurement case has shown that the measurement system can produce reliable measurement information from long-term measurements. The measurement nodes that collected data in that case, remained maintenance-free during the whole long term experiment.

For future work, the idea is to improve the adaptivity of the wireless strain nodes to enable the use of different strain gauges without having to modify the hardware. The raw-data calculation has been programmed to include more effective filtering and averaging to reduce noise from the results. For example, filtering that is based on confidence intervals has been studied. Energy consumption is always an issue with WSNs, and that is also one aspect that we still can improve in the CiNetStrain system.

In summary, we have discussed the main topics in the design and implementing of a wireless strain gauge measurement system. We have also shown the calibration and energy consumption of the strain nodes and performed an evaluation of the systems suitability for PVC-covered hall measurements.

\section{REFERENCES}

[1] Y. Chen, J. Chiang, H. Chu, P. Huang, A. Tsui, "Sensor-Assisted WIFI Indoor Location System for Adapting to Environmental Dynamics," in Proceedings of the 8th ACM Symposium on Modeling, Analysis and Simulation of Wireless and Mobile Systems, pp. 10-13. Montreal, Quebec, Canada October 2005

[2] I. Hakala, J. Ihalainen, I. Kivelä, M. Tikkakoski, "Evaluation of Environmental Wireless Sensor Network - Case Foxhouse," in International Journal on Advances in Networks and Services, 3(1\&2), pp. 22-32, September, 2010

[3] Y. Hu, D. Li, X. He, T. Sun, Y. Han, "The Implementation of Wireless Sensor Network Visualization Platform based on Wetland Monitoring," in Second International Conference on Intelligent Networks and Intelligent Systems, 2009

[4] Best-Hall Company information, Available at: http://www.besthall.com/en/company/

[5] S. Arms, C. P. Townsend, "Wireless Strain Measurement Systems Applications \& Solutions," in NSF-ESF Joint Conference on Structural Health Monitoring, pp. 3-5. Strasbourg, France October 2003

[6] P. Bielen, M. Lossie, D. Vandepitte, "A Low Cost Wireless Multichannel Measurement System for Strain Gauges," in PROCEEDINGS OF ISMA2002, vol. 2, pp. 663-670, 2002

[7] L. Jian, M. Lishchynska, K. Delaney, "Distributed Adaptive Networked System for Strain Mapping," in Third International Conference on Mobile Ubiquitous Computing, Systems, Services and Technologies UBICOMM '090, pp. 71-76, 2009

[8] MicroStrain Inc. "User Manual Version 4.0.2 SG-Link ${ }^{\circledR}$ Wireless Strain Node," MicroStrain Inc., 459 Hurricane Lane, Suite 102, Williston, VT 05495, July, 2011

[9] I. Hakala, M. Tikkakoski, "From vertical to horizontal architecture: a cross-layer implementation in a sensor network node," in Proceedings of the first international conference on Integrated internet ad hoc and sensor networks, 138(6), 2006

[10] I. Kivelä, C. Gao, J. Luomala, I. Hakala, "Design of Noise Measurement Sensor Network: Networking and Communication Part," in The Fifth International Conference on Sensor Technologies and Applications, SENSORCOMM, pp. 280-287. August, 2011

[11] NXP Laboratories, "Data Sheet: JN5148-001-Myy JenNet, ZigBee PRO and IEEE802.15.4 Module, CHIPCON," Available at: http://www.jennic.com/files/support_files/JN-DS-JN5148MO-1v4.pdf.

[12] T. Hongell, J. Ihalainen, I. Hakala, "CiNetStrain - Wireless Strain Gauge Network - Calibration and Reliability Measurements," in J. Zheng et al. (Eds.): Adhocnets 2012, LNICST 111, pp. 275-288, 2013

[13] M. Calle, J. Kabara, "Measuring Energy Consumption in Wireless Sensor Networks Using GSP," in IEEE 17th International Symposium on Personal, Indoor and Mobile Radio Communications, pp. 280-287, 2006

[14] Vishay Precision Group, "Model D4 Data Acquisition Conditioner Instruction Manual Version 1.10," Micro-Measurements, P.O. Box 27777 Raleigh, NC 27611 USA. April, 2011

[15] KYOWA, "Strain Gages for General Stress Measurement," Available at: http://www.kyowaei.co.jp/eng/content/download/4302/56924/file/KFG_eng.pdf 